\title{
Disinhibition of Perigeniculate Reticular Neurons Following Chronic Ablation of the Visual Cortex in Rats
}

\author{
Ichiji Sumitomo, Michio Sugitani and Kitsuya Iwama \\ Department of Neurophysiology, Institute of Higher Nervous \\ Activity, Osaka University Medical School, Kita-ku, Osaka 530
}

Sumtromo, I., Sugrtan, M. and Iwama, K. Disinhibition of Perigeniculate Reticular Neurons Following Chronic Ablation of the Visual Cortex in Rats. Tohoku J. exp. Med., 1977, 122 (4), 321-329 _ Effects of chronic ablation of the visual cortex (VC) were studied in the perigeniculate reticular neurons (PGR neurons) which were located in the thalamic reticular nucleus immediately adjacent to the dorsal nucleus of the lateral geniculate body and identified as the I-cells of Burke and Sefton. In rats with the intact VC the PGR neurons responded to single shock stimulation of the optic tract (OT) with bursts of spike spaced regularly. During the inter-burst period the neurons were inhibited, indicating that except for the primary spike burst, others were postinhibitory rebound excitation. In the VC-ablated PGR neurons there were no changes in the primary spike burst, but the remaining ones were very weak or sometimes missing, suggesting that the inhibition was poorly developed. With double shock stimulation of OT it was established that after showing the primary spike burst, the VC-ablated PGR neurons suffered a less intense inhibition than control. To a diffuse, sustained illumination, the normal PGR neurons showed on- and offresponses, whereas the VC-ablated ones were tonically activated during the presence of illumination. These findings were taken as indicating that the inhibitory mechanism for the PGR neurons were made less active after the VC had been ablated chronically. — perigeniculate reticular neuron; I-cells; lateral geniculate body; ablation of visual cortex; rat

The cells which are referred to as "perigeniculate reticular neuron (PGR suron)" in this paper are those of the thalamic reticular nucleus which are located ose to the dorsal nucleus of the lateral geniculate body $\left(\mathrm{LGB}_{\mathrm{d}}\right)$ and activated by sual impulses. This species of cells were first described by Burke and Sefton $966 \mathrm{a}, \mathrm{b}, \mathrm{c}$ ) with the term "I-cell" and assumed to be inhibitory interneurons of ${ } \mathrm{B}_{\mathrm{d}}$. Later several workers confirmed observations of Burke and Sefton in rats umitomo et al. 1969; Fukuda and Iwama 1971; Sumitomo 1974) and supported eir assumption. Furthermore, the idea of Burke and Sefton that intrageniculate hibitory interneurons would be like their I-cells was extended to the cat LGB $_{d}$ uzuki and Kato 1966; Sakakura 1968; Ono and Noell 1972; Levick et al. 1972).

The reason for introducing a new terminology "PGR neuron" for Burke and fton's I-cell is derived from our previous experiment; the neurons corresponding

Received for publication, January $31,1977$. 
to Burke and Sefton's I-cells were located not in $\mathrm{LGB}_{\mathrm{d}}$ but in a portion of the thalamic reticular nucleus immediately adjacent to it rostrally and medially (Sumitomo, Nakamura and Iwama 1976). In that experiment it was demonstrated that when a cluster of $P G R$ neurons were stimulated with a single electric pulse, the $\mathrm{LGB}_{\mathrm{d}}$ relay cells suffered a long-lasting inhibition and that after an electrolytic lesion was placed in the region containing the PGR neurons, the post-excitatory recovery of the $L G B_{d}$ relay cells was accelerated as if they had been disinhibited; these findings were taken as evidence that the PGR neurons are an inhibitory element for the $L_{G B}$ relay cells. Though this conclusion is not different from the original idea of Burke and Sefton, it was based upon more substantial evidence than before.

The purpose of the present paper is to show that the relation between the PGR neurons and the $\mathrm{LGB}_{\mathrm{d}}$ relay cells seems to be more complex, because it is now known that the $\mathrm{LGB}_{\mathrm{d}}$ relay cells exert inhibition upon the major group of the PGR neurons. As will be described below, this conclusion is based upon studies to see how responsiveness of the PGR neurons to visual impulses is altered after the visual cortex (VC) has been ablated chronically.

\section{METHODS}

Rats, weighing $200-300 \mathrm{~g}$, were used. They were subjected to preliminary surgery for ablation of VC. The cortical region of area 17, 18 and 18a of Krieg (1946) was ablated on one side in the way as described in a previous paper (Sumitomo, Iwama and Nakamura 1976). After more than 2 weeks were allowed for recovery, the animals were subjected to acute experiments. Each animal, anesthetized with urethane $(1 \mathrm{~g} / \mathrm{kg}$, i.p.), was fixed to a stereotaxic apparatus. A bipolar electrode, made of two insulated wires aligned singly with bared tips about $1.5 \mathrm{~mm}$ apart, was introduced into the optic chiasm to stimulate the optic tract (OT). Photic stimulation of PGR neurons was made by turning on and off a diffuse light before the eye. This was accomplished by means of a glow modulator tube (Sylvania R1131C) which was lit with a direct current of $60 \mathrm{~mA}$ and held close to the eye contralateral to the recording side.

Extracellular discharges of single PGR neurons were recorded with glass pipette microelectrodes filled with $3 \mathrm{M} \mathrm{KCl}$. The PGR neurons recorded from the VC-intact side served as control against those from the VC-ablated side.

\section{RESULTS}

In a previous study (Sumitomo, Iwama and Nakamura 1976) it was established that though most of the PGR neurons were bombarded by visual impulses monosynaptically, some were disynaptically via axon collaterals of the $\mathrm{LGB}_{\mathrm{d}}$ relay cells. In rats whose $V C$ had been ablated chronically, the $L_{G B}$ relay cells were inactive because of retrograde degeneration, so that only the monosynaptic PGR neurons could be recorded; the disynaptic PGR neurons were almost entirely missing. Since this was confirmed in the present experiments, the data which will be described below as showing the effects of chronic ablation of VC are those obtained from the monosynaptic PGR neurons. Correspondingly to this, only those data from the VC-intact side which were obtained from the monosynaptic PGR neurons were used as control. The PGR neurons were identified as monosynaptic 
A

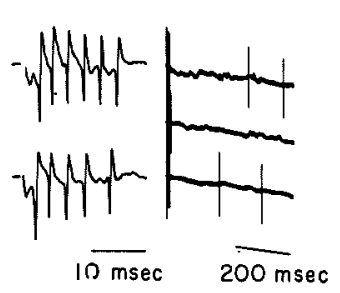

B

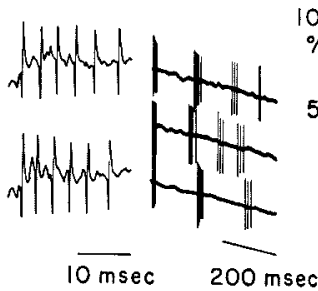

C

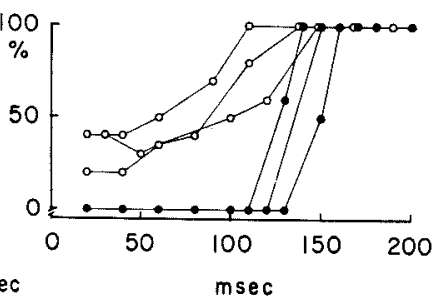

Fig. 1. A and B, responses of PGR neurons to single shock stimulation of OT. Units were from VC-ablated side in A and from VC-intact side in B. Two columns of records were made with different sweep speeds. In each case sweeps were consecutive. $\mathrm{C}$, post-excitatory recovery curves of $\mathrm{PGR}$ neurons determined by double shock stimulation of OT. Three units were sampled each from $\mathrm{VC}$-intact (filled circles) and -ablated sides (open circles). Abscissae, intervals between the first and second shocks of OT. Ordinates, response probabilities to the second shock.

when their response latencies to single OT stimuli were shorter than $3.5 \mathrm{msec}$ 'Sumitomo, Iwama and Nakamura 1976). In what follows, however, unless any specification is needed, the monosynaptic PGR neurons are simply referred to as she PGR neurons.

Responses to electrical stimulation of $O T$. A total of 28 PGR neurons were recorded rom the VC-intact side of several operated rats. Their response patterns to OT stimulation were in good agreement with what has been observed in intact rats 'Burke and Sefton 1966a, b, c; Sumitomo et al. 1969; Fukuda and Iwama 1973; Jumitomo 1974). Sample records are shown in Fig. $1 \mathrm{~B}$; following single OT shocks, several groups of spikes are elicited being separated by fairly regular ntervals. The earliest spike burst, which occurs with latencies of 2-6 msec, is sscribed to the primary excitatory effect of OT impulses (Fig. $1 \mathrm{~B}$, left). It is :ollowed by the second burst of spikes after a silent period of more than 100 nsec, and thereafter a similar sequence of excitation and silence ensues (Fig. 1 B, :ight). As will be described below, there is evidence that during the silent period Jetween the first and the second spike burst the neurons are inhibited. Therefore, $t$ is reasonable to regard the second spike burst as a rebound excitation consequent upon a removal of the inhibition following the primary excitation. Probably the same may be true for the remaining spike bursts.

From the VC-ablated side a total of 24 PGR neurons were recorded. As far as ;he earliest spike burst to OT shocks was concerned, the PGR neurons of the VCzblated side did not show any abnormality (Fig. $1 \mathrm{~A}$, left); the number of spikes sontained and the latency of the very first spike were in the same range as observed $\mathrm{n}$ the control PGR neurons. Thus, it is said that the transmission of the Jrimary excitatory effect of visual impulses to the PGR neurons is not affected by shronic ablation of VC. However, there was a change in the bursting activity collowing the primary excitation; only one spike or two at most occurred as a esidue of the bursting activity or sometimes there were no spikes at all (Fig. 1 A, 
right). This is interpreted as indicating that in the PGR neurons of the VC-ablated side the inhibition which is to follow the primary excitation is not produced with a sufficient intensity so that the rebound excitation is weak or almost absent.

Comparison of the post-excitatory recovery of PGR neurons between the VC-intact and -ablated sides. In order to give a more concrete basis to the above-mentioned interpretation, experiments were made to measure the recovery process of PGR neurons which follows the primary excitation; OT was stimulated with double shocks and responsiveness to the second shock was examined at varying intervals after the first shock. The stimulus intensity, set equal for the two shocks, was twice the threshold of the primary excitation of individual PGR neurons. As the index of responsiveness, the probability for occurrence of the primary excitation to the second shock was determined from ten successive trials.

The data from three typical PGR neurons of the VC-intact side are summarized in Fig. $1 \mathrm{C}$ (filled circles); the cells' responsiveness to the second shock was completely suppressed up to $110-130 \mathrm{msec}$ after the first shock, and thereafter it promptly resumed the level of $100 \%$. This agrees well with previous data of Burke and Sefton (1966b). In the PGR neurons of the VC-ablated side, however, depression of the responsiveness after the primary excitation was not so profound as in control. In Fig. $1 \mathrm{C}$, this is shown with the data from three neurons (open circles); even in the phase of maximal depression the neurons retained the responsiveness of 20 $40 \%$ and they soon started to recover towards the level of $100 \%$. A conclusion is thus reached that in the PGR neurons of the VC-intact side there develops a profound inhibition following the primary excitation, whereas in the VCablated side the corresponding inhibition is considerably weakened. These findings justify our presumption that the strength of the bursting activity following the primary excitation is dependent upon whether the inhibition is well-developed or not.

There is one additional finding that was interpretable along the same line as above. It is concerned with responsiveness of the PGR neurons to repetitive stimulation of OT. When OT is stimulated repetitively, say at $10 \mathrm{~Hz}$, the PGR neurons show only the primary excitation. If the stimulus frequency is gradually increased beyond that level, it is seen that the primary spike burst is cut from behind and finally a frequency is reached for which one stimulus elicits only one spike and no spikes are elicited with stimulus frequencies higher than it. Such critical frequency of OT stimulation was below $20 \mathrm{~Hz}$ in most of the PGR neurons of the VC-intact side. With the PGR neurons of the VC-ablated side it was also true that as the frequency of OT stimulation was increased, the number of spikes elicited by one stimulus was decreased and finally the critical frequency was reached yielding the response pattern of one-stimulus-to-one-spike. However, the VC-ablated PGR neurons were distinguished from control in that the critical frequency of OT stimulation was much higher than in control; it ranged from $80-$ $100 \mathrm{~Hz}$. 
One possible interpretation for the failure of high-frequency stimulation of OT , excite the PGR neurons would be that the inhibitory effect produced by dividual OT stimuli accumulates to the extent to which the primary excitatory fect would be reduced or nullified. Then, the above results would be taken , indicate that the inhibitory effect by OT stimuli accumulated in the PGR neurons ore easily in the VC-intact side than in the VC-ablated one. This is consistent ith what has been described above; the inhibitory mechanism of the PGR neurons as been made less active by chronic ablation of VC.

'esponses of VC-intact PGR neurons to photic stimulation. Sefton and Bruce (1971) sported that the PGR neurons show on- and off-responses to a diffuse, sustained lumination. In the present experiment this was confirmed as exemplified by unit ${ }_{3}$ in Fig. 2. This unit responded to OT shocks with well-developed spike bursts ecords a and b), satisfying the criteria for the PGR neuron of the VC-intact side. Ipon turning on a diffuse light, spikes were bursted transiently, but thereafter the nit remained silent until the off-response appeared. The latter occurred with a itency of about $0.1 \mathrm{sec}$ and lasted for less than $1 \mathrm{sec}$ (record c).

It is without doubt that the on-response to photic stimulation represents he primary excitatory effect of visual impulses as is the case with the earliest pike burst caused by electrical stimulation of OT. In the latter case the primary xcitation was proved to be followed by the inhibitory phase. The same would be
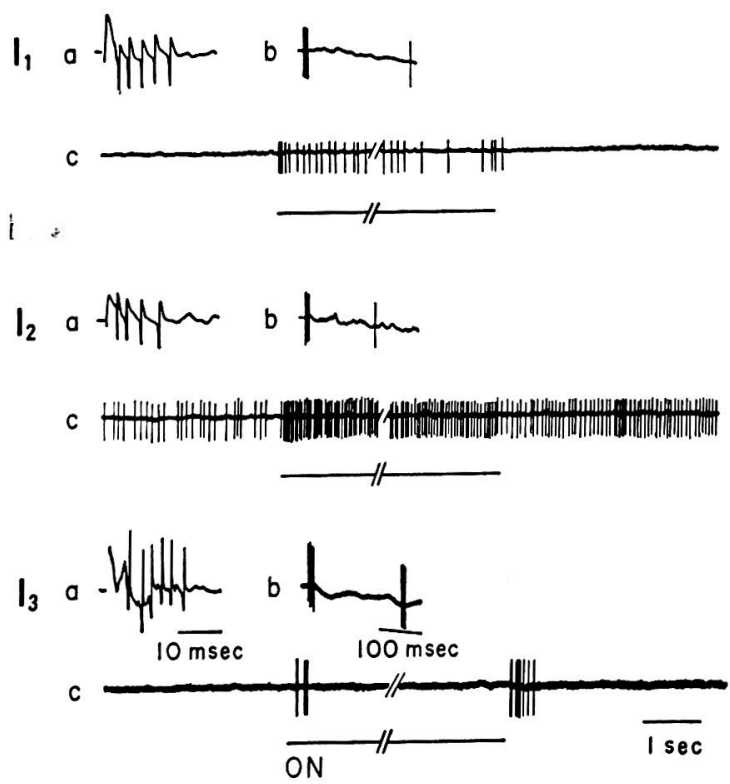

Fig. 2. $I_{1}$ and $I_{2}$, different units from VC-ablated side. $I_{3}$, a unit from VC-intact side. In each frame, $a$ and $b$ are responses to single shock stimulation of OT, displayed with different sweep speeds, and c, a response to a diffuse illumination lasting more than 5 sec. 


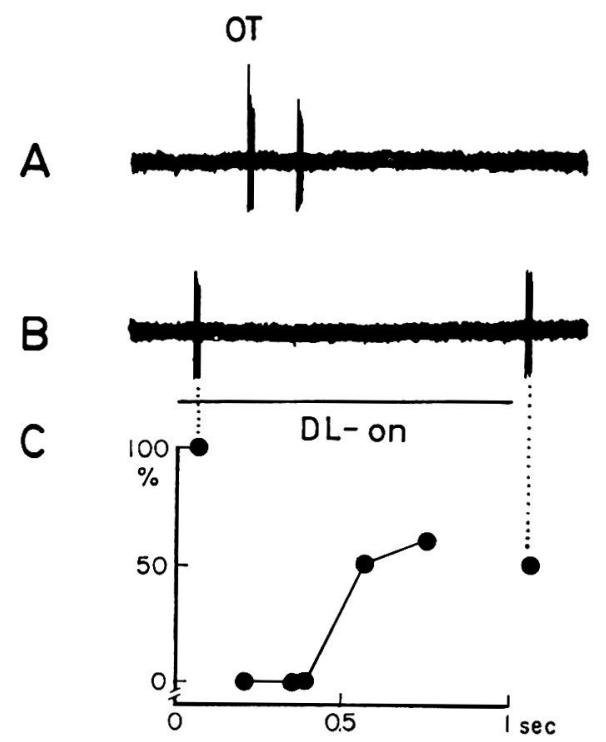

Fig. 3. Inhibition of a normal PGR neuron during the presence of continuous illumination. $A$, response to a testing OT shock. B, response to a diffuse illumination lasting 1 sec (DL-on). C, response probabilities to testing OT shocks as a function of time after the onset of illumination. The right- and left-most circles indicate the probabilities for appearance of the on-and off-responses.

true in the photically evoked response, i.e. during the silent period between the on- and off-responses the PGR neurons would be in an inhibited state. This was evidenced by testing responsiveness of PGR neurons to OT shocks. An example of the data is shown in Fig. 3. Record A shows responses of a PGR neuron to a testing OT shock. This cell responded to single OT stimuli with two bursts of spikes spaced by about 0.1 sec. In $\mathrm{B}$, the cell was stimulated by a diffuse light lasting 1 sec. Although the on-response was elicited with the probability of $100 \%$, the off-response failed to appear in half the trials. During the presence of continuous illumination, OT was stimulated at varying intervals after the on-response and the probabilities for appearance of the OT-induced primary excitation were determined. As shown in C, the cell could not be fired by a testing OT shock untill 0.3 sec after the on-response. Thereafter, its responsiveness was gradually recovered, but it remained around $60 \%$ of control towards the end of illumination.

From these results it is concluded that after having shown the on-response to the onset of continuous illumination, the PGR neurons are brought to the inhibited state, though the strength of inhibition tends to decrease gradually. This conclusion leads us to an assumption that the off-response would be a rebound excitation resulting from a removal of the inhibition maintained during the presence of continuous illumination.

Responses of VC-ablated PGR neurons to photic stimulation. With regard to responsiveness to a diffuse, sustained illumination, the PGR neurons of the VC-ablated 
ide were again distinguished from control. Two sample units are given in Fig. 2 $I_{1}$ and $I_{2}$ ). Insets $a$ and $b$ show that their response patterns to OT shocks were sonsistent with the above-described characteristics of the VC-ablated PGR neurons. Unit $I_{1}$ had no spontaneous discharges before photic stimulation and was continuously xctivated after turning on a diffuse light, though the discharge rate was gradually lecreased towards the end of illumination. Unit $I_{2}$ was considerably excited during lark and increased the discharge rate enormously during the presence of illumination. The discharges were definitely reduced after the stimulus was withdrawn. It seems that the cell was again excited towards the end of record. However, this cannot be taken as the off-response, because its latency, measured from the end of illumination, was too large to be taken as indicating occurrence of an enhanced off-response.

To sum up, chronic ablation of VC alters the response pattern of the PGR neurons to a sustained illumination; they are excited continuously during the presence of illumination. It looks as if the transient on-response of the normal PGR neurons has been altered so as to last long. As deseribed in a previous section, it is surmised that in the VC-ablated condition the PGR neurons are more or less freed from inhibition. In such condition the PGR neurons would tend to discharge continuously as long as an illumination is maintained. The off-response, which is regarded as a post-inhibitory rebound excitation, would be expected to decrease in the disinhibited PGR neurons. Though this point could not be proved positively, the present results are not against it; in none of the PGR neurons from the VC-ablated side one could observe that the off-response was made enhanced or long-lasting.

\section{Discussion}

As described in a previous section, the PGR neurons recorded from the VCablated side are almost exclusively those connected to OT fibers monosynaptically. In the VC-intact side recordings were limited to the monosynaptic PGR neurons to facilitate a comparison with the VC-ablated side. The data assembled indicate that in the rat with the intact VC, visual impulses first excite the monosynaptic PGR neurons and then bring them to an inhibited state which is terminated by a rebound excitation, and this inhibition is weakened or almost nullified after $\mathrm{VC}$ has been ablated chronically. This latter fact is most strongly supported by the finding that in the VC-ablated condition the monosynaptic PGR neurons respond to a continuous illumination with a pattern of tonic discharges which is never seen in the VC-intact condition. Though the normal function of the VC is a requisite for the inhibition of the monosynaptic PGR neurons, it is unlikely that the inhibition is produced by visual impulses which are transmitted to $\mathrm{VC}$ and reflected therefrom to the target neurons, because acute ablation of $\mathrm{VC}$ does not yield any sign suggesting a deterioration of the inhibitory mechanism for the monosynaptic PGR neurons. The structure responsible for such mechanism should be the one which loses its function as the chronic effects of $\mathrm{VC}$ ablation become manifest. 
We presume that a most likely candidate is $\mathrm{LGB}_{\mathrm{d}}$. Its relay cells have been proved to transmit visual impulses to the PGR neurons of the disynaptic type. If the disynaptic PGR neurons were assumed to be inhibitory neurons for the monosynaptic ones, the present results are interpreted as follows. In the condition of intact VC, visual impulses excite the monosynaptic PGR neurons and immediately following it, inhibit them by activating the disynaptic PGR neurons via the route of axon collateral of the $\mathrm{LGB}_{\mathrm{d}}$ relay cells. The primary excitation of the monosynaptic PGR neurons can occur even in the VC-ablated condition. However, in this condition, the inhibition following the primary excitation is lessened very much, because the $\mathrm{LGB}_{\mathrm{d}}$ relay cells have been made inactive due to retrograde degeneration and consequently the number of activated disynaptic PGR neurons is greatly reduced or almost none. This results in disinhibition of the monosynaptic PGR neurons.

So far we have discussed the inhibitory action originating in the disynaptic PGR neurons and affecting the monosynaptic ones. Despite such a functional difference between the two types of PGR neurons, these cannot be differentiated in other characteristics. For example, when bombarded by visual impulses in the VC-intact condition, the disynaptic PGR neurons are first excited and then inhibited in the same manner as are the monosynaptic ones. With regard to the inhibition of the disynaptic PGR neurons, no data are available suggesting the structures responsible for it. Further experiments are needed to clarify this point.

\section{References}

1) Burke, W. \& Sefton, A.J. (1966a) Discharge patterns of principal cells and interneurons in lateral geniculate nucleus of rat. J. Physiol. (Lond.), 187, 201-212.

2) Burke, W. \& Sefton, A.J. (1966b) Recovery of responsivneness of cells of lateral geniculate nucleus of rat. J. Physiol. (Lond.), 187, 213-229.

3) Burke, W. \& Sefton, A.J. (1966c) Inhibitory mechanism in lateral geniculate nucleus of rat. J. Physiol. (Lond.), 187, 231-241.

4) Fukuda, Y. \& Iwama, K. (1971) Reticular inhibition of internuncial cells in the rat lateral geniculate body. Brain Res., 35, 107-118.

5) Krieg, W.J.S. (1946) Connections of the cerebral cortex. I. The albino rat. A: Topography of the cortical areas; and B: Structures of the occipital area. J. comp. Neurol., 84, 221-323.

6) Levick, W.R., Cleland, B.G. \& Dubin, M.W. (1972) Lateral geniculate neurons of cat: Retinal inputs and physiology. Invest. Opthalamol., 11, 302-311.

7) Ono, T. \& Noell, W.K. (1972) Characteristics of P- and I-cells of cat's lateral geniculate body. Vision Res., 13, 639-646.

8) Sakakura, H. (1968) Spontaneous and evoked unitary activities of cat lateral geniculate neurons in sleep and wakefulness. Jap. J. Physiol., 18, 23-42.

9) Sefton, A.J. \& Bruce, I.S.C. (1971) Properties of cells in the lateral geniculate nucleus. Vision Res., (Suppl. 3), pp. 239-252.

10) Sumitomo, I. (1974) Effects of thalamic midline nuclei stimulation upon lateral geniculate transmission in rats: Inhibition of principal cells and excitation of internuncial cells. Tohoku J. exp. Med., 114, 227-240.

11) Sumitomo, I., Ide, K., Iwama, K. \& Arikuni, T. (1969) Conduction velocity of optic nerve fibers innervating lateral geniculate body and superior colliculus in 
the rat. Exp. Neurol., 25, 378-392.

12) Sumitomo, I., Iwama, K. \& Nakamura, M. (1976) Optic nerve innervation of socalled interneurons of the rat lateral geniculate body. Tohoku J. exp. Med., 119, 149-158.

13) Sumitomo, I., Nakamura, M. \& Iwama, K. (1976) Location and function of the socalled interneurons of rat lateral geniculate body. Exp. Neurol., 51, 110-123.

14) Suzuki, H. \& Kato, E. (1966) Binocular interaction at cat's lateral geniculate body. J. Neurophysiol., 29, 909-919. 